\title{
Design \& Development of High Data Rate Orthogonal Frequency Division Multiplexing System Using FFTs
}

\author{
Shakti khatri ${ }^{1}$, Er. Neeraj Mann ${ }^{2}$ \\ Electronics \& Communication Engg Department, P.M College of Engineering, Sonepat ${ }^{1,2}$
}

\begin{abstract}
In recent years, Orthogonal Frequency Division Multiplexing (OFDM) has been successfully used in terrestrial digital video broadcasting and showed it is a strong candidate for the modulation technique of future wireless systems. This paper is concerned with how well OFDM performs in digital broadcasting when transmitted over an Additive White Gaussian Noise (AWGN) channel only. The main objective of this work is to design an OFDM system for digital broadcasting standard. In this, it uses $2 \mathrm{k}$ mode and $8 \mathrm{k}$ mode system. OFDM is based on FFT based subcarriers. The main problem in OFDM is PAPR value. So, in this work, it also improves the value of PAPR under different modulation formats. The Digital Video Broadcasting Project (DVB) is a multinational initiative to standardize digital broadcasting worldwide. The purpose of this paper is to provide a simulation of the process involved in the generation and reception of an OFDM signal in a physical channel and to provide a description of each of the steps involved.
\end{abstract}

Keywords: OFDM System, Digital Broadcasting, QAM modulation, Demodulation etc.

\section{INTRODUCTION}

As high data-rate communication systems tend to play an increasing role in our daily life, more and more research is being done on them. The vast number of applications involving high data-rate systems made it essential to achieve the best possible performance with the lowest possible cost. Normally, these high data-rate system suffer from the presence of multipath channels. This leads to the fact that the receiver is no longer able to distinguish the different symbols due to the delays occurring in each separate copy of the transmitted signal that arrives at the receiver. Thus complex equalizers are needed at the receiver end [1].

A solution to that problem is the use of OFDM. OFDM stands for Orthogonal Frequency Division Multiplexing. It is a digital modulation technology used in many of the high data-rate systems such as DAB (Digital Audio Broadcast), DVB-T (Digital Video Broadcast - Terrestrial), high speed telephone line communication such as XDSL (Digital Subscriber Line), and WLAN (Wireless Local Area Network) [2].

One OFDM symbol is a set of a large number of orthogonal waves. The transmission is divided into smaller sub-bands, each having a low separate rate and is insensitive to dispersive multipath channels. Thus complex equalizers are not needed and a lower complexity is accomplished. Inter-symbol Interference (ISI) is avoided by introducing a guard interval between successive symbols. This is the price to be payed for simple receiver structures. Whereas OFDM solves the above mentioned problem, it introduces new problems itself. By using high numbers of narrow sub-carriers, the system becomes very sensitive to time and frequency offsets and thus a precise synchronization is needed [3].

Synchronization remains as one of the most important factors to think of while using OFDM. Many algorithms have been developed and analyzed in the literature, but still in the domain needs a lot to be done. This thesis aims at suggesting best synchronization solutions depending on priorities and trade-offs between cost (complexity), timeto-market and performance. Therefore, different synchronization techniques and algorithms are studied, analyzed, simulated and optimized leading to a hardware implementation [4].

The first proposal to use OFDM for transmission appeared in 1966 and was introduced by Robert W. Chang [10]. This document presents the way to implement OFDM and also explains its main concept. OFDM symbols are created by filtering the signal and then multiplying the outputs by different frequencies. Thus, the subcarriers are orthogonally created, they overlap between each other and they are band-limited. Therefore, the spectra are produced without causing ICI and ISI. This method supposed a huge revolution in the communication world and in 1969 appeared the Discrete Fourier Transform (DFT) as a way to generate the orthogonal subcarriers [2]. Later on, in the 80 's appeared the concept of CP whose purpose was to resolve the channel dispersion-induced ICI and ISI.

In 1995 Telatar and Foschini studied OFDM, for multiple antenna systems opening a new investigation area. OFDM also began to be considered in wireless communications after the publication of Cimini of Bell labs in 1985. While in 1987 Lassalle and Alard proposed OFDM for digital broadcasting for mobile receivers. This article explains the benefits of using OFDM to overcome the adverse effects of severe multi-path propagation. Cioffi and others at Stanford demonstrated in 1990 the potential to apply OFDM in wire line communications [16], designing a Discrete Multi Tone (DMT) transceiver for High-Bit-Rate Digital Subscriber Line (HDSL). So with the advancement of powerful silicon DSP technology, OFDM triumphed in 
a broad range of applications such as the RF domain from digital audio/video broadcasting (DAB/DVB) to wireless local area networks (LANs). OFDM has been recently applied to optical communications. In 2001 appeared the first paper based on OFDM for optical wireless and in 2005 it was used in optical fiber communication systems [5].

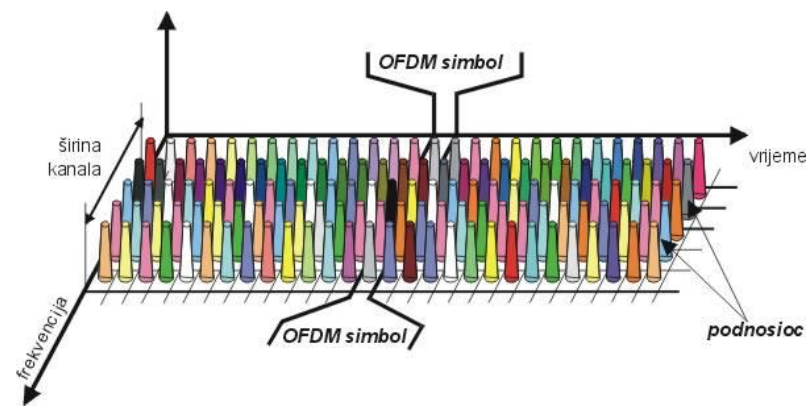

Figure 1: OFDM Pattern

OFDM has emerged as a leading modulation technique and in the optical domain. It is also used in wireless and wire-line applications and in almost every major communication standards. The use of OFDM in optical communications mitigates transmission impairments and, at the same time, provides high-data rate transmission across dispersive optical media. The progress in Digital Signal Processing (DSP) technology can make processing at optical data rates feasible. O-OFDM introduces spectral efficiency and tolerance to impairments such as chromatic dispersion (CD) and polarization mode dispersion (PMD) to the system. It belongs to a broader class of MultiCarrier Modulation (MCM) in which data information is carried over many lower rate subcarriers. The subcarriers are orthogonal to each other, and their spectra can overlap. This results in a very high spectral efficiency [5].

The insertion of a Cyclic Prefix (CP) makes OFDM an effective solution to Inter-Symbol Interference (ISI) and Inter-Carrier Interference (ICI), caused by a dispersive channel that can degrade the performance of the system. This CP consists of an identical copy of the first samples of the frame that are added at the end of it, implying an increase of the signal bandwidth. The signal processing in the OFDM transmitter/receiver is based on the FFT to implement the OFDM modulation/demodulation. So the symbols can be generated in a very computationally efficient way. Other transforms are also suitable to generate the OFDM symbols. This is the case of the Fast Hartley Transform (FHT) that has recently been introduced in Intensity-Modulation (IM)/Direct-Detection (DD) systems because a simpler transmission system can be achieved with real processing.

The paper is ordered as follows. In section II, it represents related work with proposed system in OFDM System. In Section III, It defines basics of OFDM technique. In Section IV, It defines proposed OFDM System. Finally, conclusion is explained in Section V.

\section{LITERATURE REVIEW}

Authors proposed the behaviour of DD-OFDM with different high order modulation formats from 4 QAM to
64 QAM. The impact of fiber nonlinearity for 4-QAM modulation format in long-haul WDM-OFDM transmission with direct detection is also investigated. Bitrate up to a factor of 3 is increased without requesting more bandwidth or higher speed components. [6]

Due to rapid growth of internet traffic and capacity requirement applications, there is urgent need for optical fiber transmission technology. Authors approved Optical OFDM as a promising technology for future high-speed transmission because of its superior tolerance to CD/PMD, high spectrum efficiency, and scalability to ever increasing data rates based on its subcarrier multiplexing technology. It presented a comprehensive survey that covers optical OFDM transmission \& OFDM based elastic optical networking. OFDM principles including its mathematical formulation, the main building blocks, and related key functions were also introduced. [7]

Some proposed ADO-OFDM, a recently developed modulation scheme for IM/DD systems. Performance of ADO-OFDM is compared with ACO-OFDM and DCOOFDM. The subcarriers in ADO-OFDM are modulated using ACO-OFDM and DCO-OFDM. Also the performance of the ADO-OFDM depends on a number of parameters including the proportion of power allocated to ACO-OFDM and DCO-OFDM, and constellations used on each component. [8]

Some presented a realistic performance analysis on IEEE 802.15.4g MR-OFDM multi-hop system in an environment with multi-PHY interference. Extensive cross-layer computer simulations are conducted to validate the accuracy of the system performance. We can do the Future work in this work in frequency-agile mechanisms such as frequency hopping operations. [9]

Some demonstrated Adapted and extended GMPLS in order to deploy a control plane for elastic/flexible optical networks with coherent optical OFDM transmission technology. The control plane protocol extensions involved in the main functional aspects have been presented and will be useful for future standardization. The integrated system in a control plane test bed emulating a 14-node Japan topology is evaluated in this paper. Different key performance indicators such as path setup latency for different assignment strategies are obtained. [10]

An ICA-based CE is presented to recover the OFDM signal in optical OFDM systems without using TSs. The ICA-based CEs are used to compensate the linear channel impairments in DDO-OFDM and PDM-CO-OFDM system. 3 types of ICA algorithms including MN, ML, MMI are applied for the purpose of channel equalization [11].

\section{DESCRIPTION OF PROPSED SYSTEM}

Orthogonal frequency division multiplexing (OFDM) is a modulation technique which belongs to a broader class of multicarrier modulation (MCM) in which the data information is carried over many lower rate subcarriers. Two of the fundamental advantages of OFDM are its robustness against channel dispersion and its ease of phase and channel estimation in a timevarying environment. OFDM is now used in most 
new and emerging broadband wired and wireless word). Each data word is mapped to one unique IQ communication systems because it is an effective location in the constellation. A large number of solution to inter-symbol interference caused by a modulation schemes are available allowing the number of dispersive channel.

The development of OFDM system can be divided into three stages, these are - Frequency division multiplexing, multicarrier communication and orthogonal frequency division multiplexing. The OFDM system can be divided into three parts mainly, transmitter, channel and receiver. The block diagram of a typical OFDM transmitter and receiver is shown in figure $4 \& 5$. The OFDM transmitter works as: The input serial data stream is first converted into many parallel data streams after passing through a serial - to - parallel converter. These parallel data streams are modulated onto the orthogonal subcarriers and changed to the time domain OFDM symbol by applying the IFFT. The OFDM signal is then converted to analog signal by applying the digital-to-analog conversion (DAC) and filtered with low pass filter.

The baseband signal thus obtained can be up converted to an appropriate RF. At the receiver, the received signal is first down converted, sampled with an ADC and then the complex form OFDM signal is demodulated by applying the FFT. The demodulated signals go through a symbol decision module. Finally, the multiple data channels are converted back to a single data stream by applying the parallel - to - serial data converter.

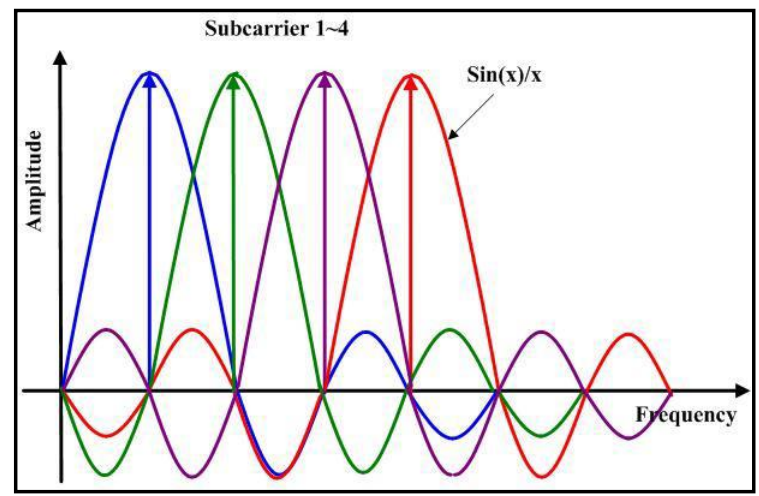

Figure 2: OFDM Spectrum in Frequency Domain [12]

The first step in the transmitter is generating theMPEG-2 transport packets. In the simulations random data have been used. By this we skip the step of randomizing the data with a scrambler. After the packets are generated they have to be adapted to the channel characteristics of the DVB-T standard. First, we convert the random bits into modulated symbols, with one of the following signal constellations: QPSK, 16-QAM, 64-QAM.

The input data stream is formatted into the word size required for transmission in each OFDM symbol. For example, for a subcarrier modulation of 4-QAM, each subcarrier carries 2 bits of data, 16-QAM has 4 bits and 64-QAM has 6 bits of data. So for a transmission using 6817 subcarriers using 4-QAM, the number of parallel symbols entering the IFFT block is 4096. The modulation scheme is a mapping of data words to a real (In phase) and imaginary (Quadrature) constellation, also known as an IQ constellation. For example, 64-QAM involves 6 bits in each symbol (data bits transmitted per subcarrier per symbol to be varied. In the receiver, the received IQ symbol de-mapped back to data words. This is called Demodulation.

The codification of the signal adds enough redundancy and protection to permit the correction of errors and to make the signal more robust. The Forward Error Correction (FEC) is used after the signal pass through the channel. This codification allows to retrieve the information transported by subcarriers that are cancelled due to the selective fading of the radio channel. At the transmitter of an OFDM system, data are apportioned in the frequency domain and an IFFT is used to modulate the data into the time domain. The FFT output data are guaranteed to be real-valued if conjugate symmetry is imposed on the input data. In the receiver, an FFT is used to recover the original data. The FFT allows an efficient implementation of modulation of data onto multiple carriers [11]. Due to the similarity between the forward and inverse transform, the same circuitry, with trivial modifications, can be used for both modulation and demodulation in a transceiver. A fundamental challenge with OFDM is that a large number of subcarriers are needed so that the transmission channel affects each subcarrier as a flat channel. This leads to an extremely complex architecture involving many oscillators and filters at both transmit and receive ends.

To reduce the effects owing to the radio channel is used the Orthogonal Frequency Division Multiplexing (OFDM). This technique consists in a multi-carrier modulation where the signal is divided in $\mathrm{N}$-flows of low speed that modules several sub-carriers. The duration of the low speed symbols are selected in a way that exceed the dispersion time including the last echo. Every subcarrier that is modulated has a zero in its spectrum at the frequency of the following sub-carrier. In such a way, they are orthogonal. To obtain this, the frequencies of every sub-carrier have to be separated the same value as the inverse of the low speed symbols' duration.

Here, the OFDM subcarriers are generated in the digital domain using IFFT. The FFT-based O-OFDM transmitter is composed of a RF OFDM transmitter and a RF-tooptical up-converter, while the receiver is composed of an optical-to-RF down-converter, and a RF OFDM receiver.

\section{Advantages of OFDM}

There are many advantages of OFDM. Some of these are -

- OFDM divides the high-speed data stream into multiple low-data-rate subcarriers and then transmit them, thereby increasing the symbol duration and reducing the ISI, thus making OFDM, a good candidate for future high-speed communication systems.

- Smooth upgrading from low-speed transmission to highspeed transmission is possible in OFDM without major changes in system design.

- OFDM gives high spectrum efficiency and capacity.

- Energy-efficient operation is possible with OFDM through adaptive modulation and dynamically switching 
INTERNATIONAL JOURNAL OF INNOVATIVE RESEARCH IN ELECTRICAL, ELECTRONICS, INSTRUMENTATION AND CONTROL ENGINEERING Vol. 3, Issue 12, December 2015

on/off specific subcarriers according to customer In Fig. below, we observe the result of this operation and bandwidth requirements and channel conditions.

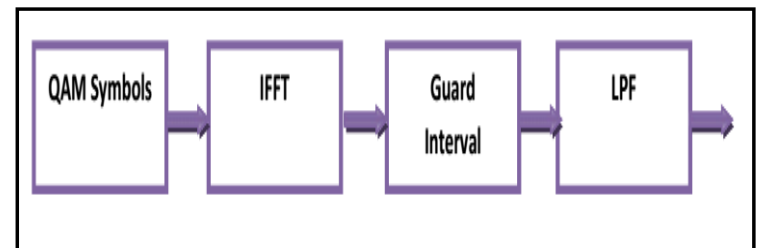

Figure 3: Generation of OFDM System

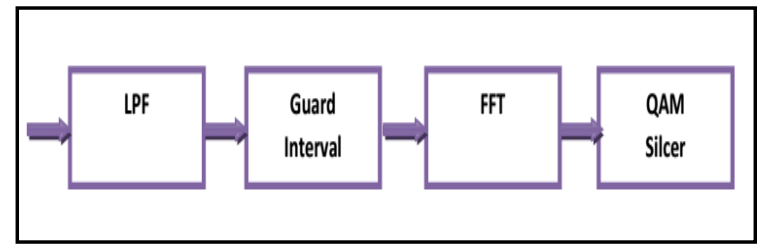

Figure 4: Reception of OFDM System

Performance Parameters: PAPR (Peak to Average Power Ratio)

An OFDM consists of a number of independently modulated subcarriers, which can give a large PAPR value when added up coherently. Large PAPR means large amplifier back-offs, which reduces power efficiency of RF amplifier and also increase the complexity of converters.

\section{RESULTS}

The first task to consider is that the OFDM spectrum is centered on fc ; i.e., subcarrier 1 is $7.612 \mathrm{MHz}$ to the left of the carrier and subcarrier 1,705 is $7.612 \mathrm{MHz}$ to the right. One simple way to achieve the centering is to use a $2 \mathrm{~N}$-IFFT [2] and T/2 as the elementary period. The figure 4.3 shows the proposed GUI of $2 \mathrm{k}$ mode of DVB system using OFDM. The elementary time period for a base band signal is taken as $\mathrm{T}$.

Here we consider a simple integer relation $\mathrm{RS}=40 / \mathrm{T}$. This integer relation gives us a frequency close to $90 \mathrm{MHZ}$. Now we design the transmitter, and for that the steps undertaken have been shown. At first we add 4,096$1,705=2,391$ zeros to the signal info at (A) to achieve over- sampling, and to center the spectrum.

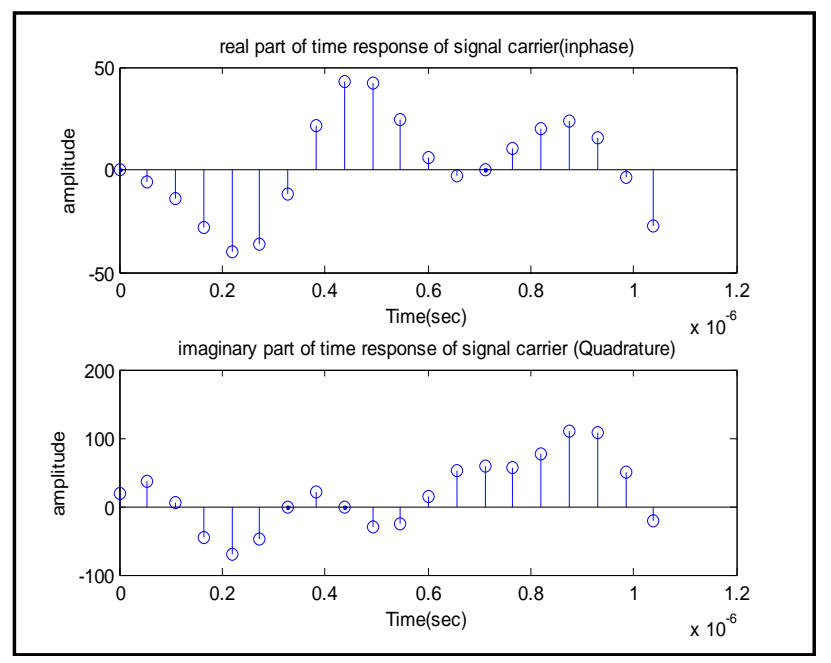

Figure 5: Carrier Signal Data in Time Domain that the signal "carriers" uses T/2 as its time period. We can also notice that "carriers" is a discrete time baseband signal. The first step is to produce a continuous-time signal and to apply a filter $\mathrm{g}(\mathrm{t})$, to the complex signal "carriers". The impulse response, or pulse shape, of $g(t)$ is shown.

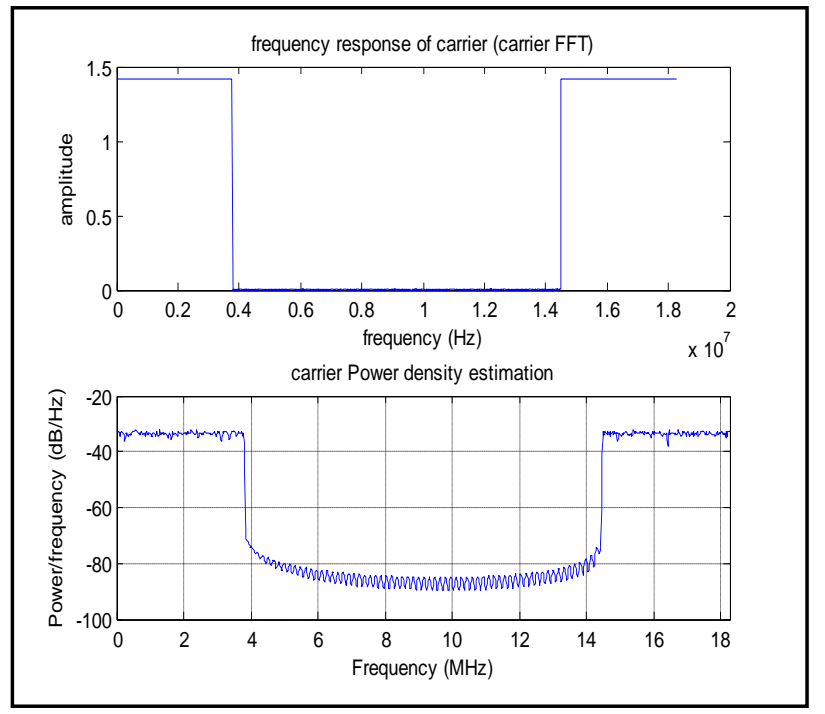

Figure 6: Carrier Signal Data in Frequency Domain

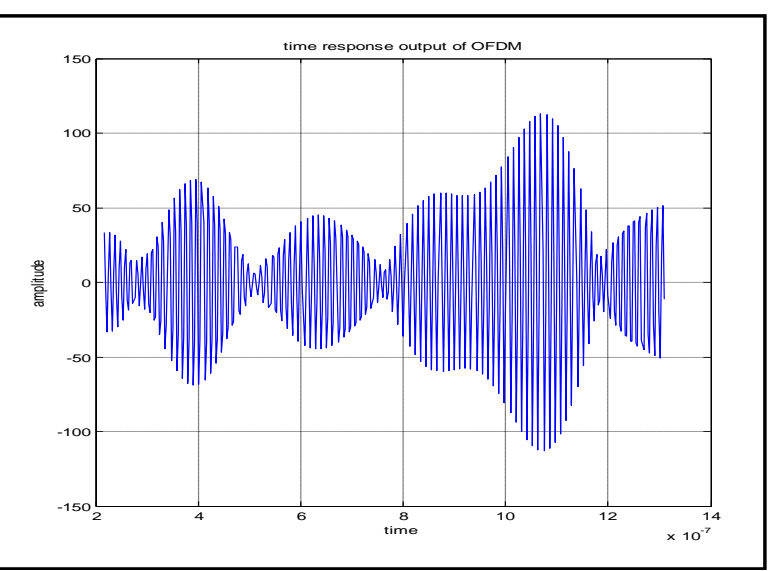

Figure 7: OFDM Output of 2KDVB System

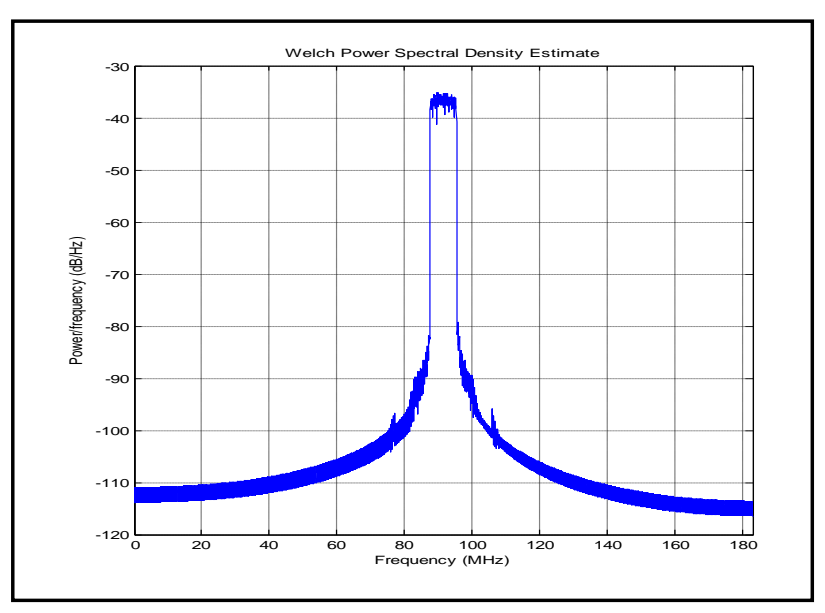

Figure 8: OFDM Power Spectra Using 2KMode 
The first thing to notice is the delay of approximately $2 \times 10-7$ produced by the filtering process. Aside of this delay, the filtering performs as expected since we are left with only the baseband spectrum. We must recall that subcarriers 853 to 1,705 are located at the right of $0 \mathrm{~Hz}$, and subcarriers 1 to 852 are to the left of $4 \mathrm{fc} \mathrm{Hz}$

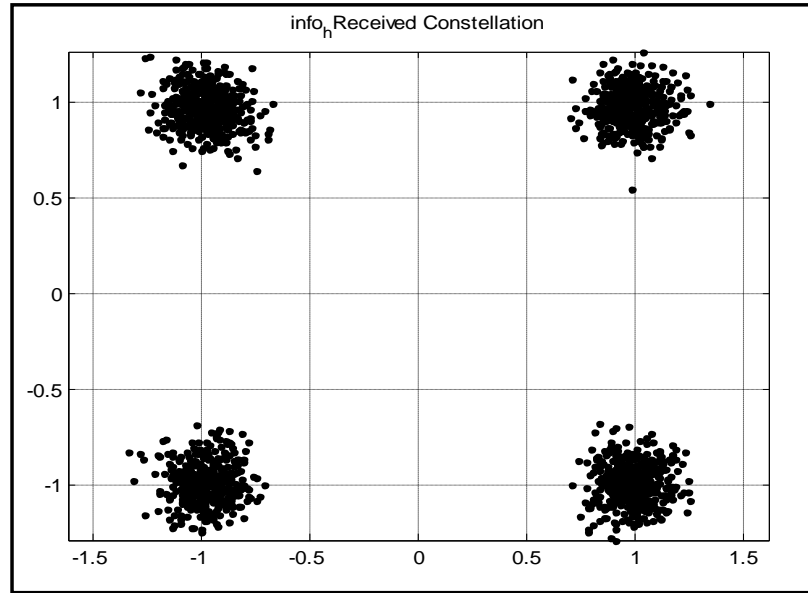

Figure 9: Received 4 QAM Constellation Plot

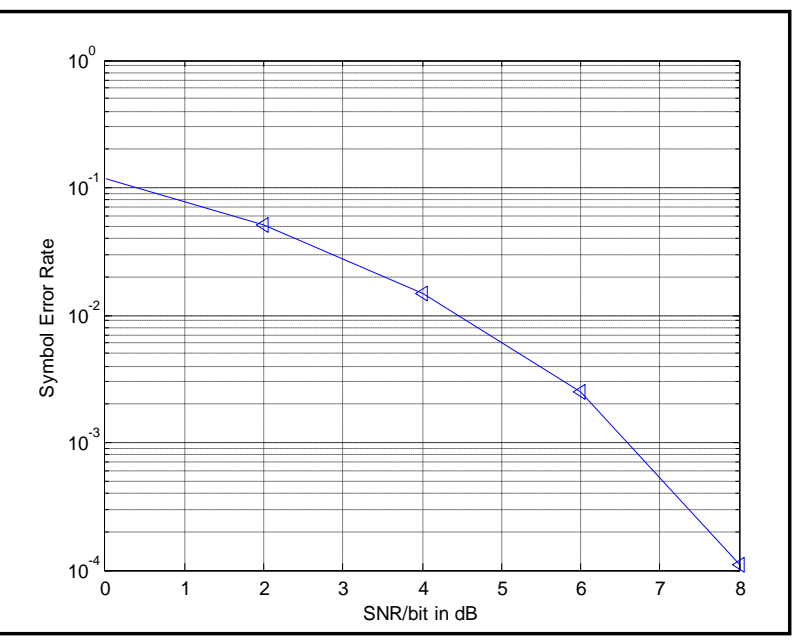

Figure 10: SER Output of 2k Mode

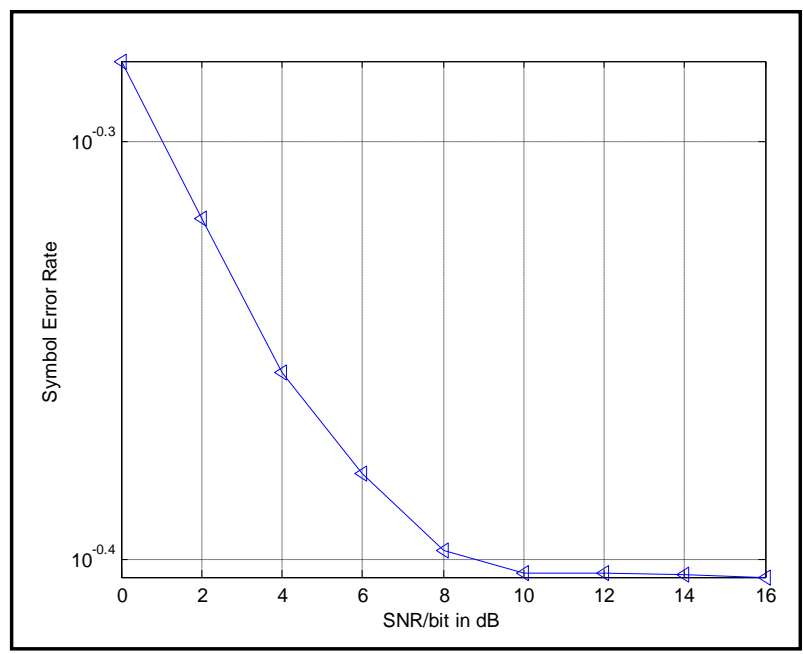

Figure 11: SER Output of 8k Mode

\section{CONCLUSION}

OFDM is a very attractive technique for multicarrier transmission and has become one of the standard choices for high - speed data transmission over a communication channel. The main objective of this work is to design an OFDM system for digital broadcasting standard. In this, it uses $2 \mathrm{k}$ mode and $8 \mathrm{k}$ mode system. OFDM is based on FFT based subcarriers. The main problem in OFDM is PAPR value. So, in this work, it also improves the value of PAPR under different modulation formats. The DVB-T system for terrestrial broadcasting is probably the most complex DVB delivery system. Originally, the DVB-T standard was created for fixed and portable reception. As expected it has proven a worldwide success. After this, different modulation formats are also compared on the basis of performance parameters. The Additive White Gaussian Noise (AWGN) corrupted the transmitted signal and this resulted in a different received 4QAM constellation than the original constellation.

\section{REFFERENCES}

[1] Bharath Umasankar, A. D. S. Jayalath, "Adaptive Performance Improvement of OFDM Radio over Fiber Systems", IEEE 2007.

[2] Xingwen Yi, William Shieh, "Phase Noise Effects on High Spectral Efficiency Coherent Optical OFDM Transmission", IEEE Journal Of Lightwave Technology, Vol. 26, No. 10, May 15, 2008.

[3] S.Jawla, R.K.Singh, "Different Modulation Formats Used In Optical Communication System" IOSR Journal of Electronics and Communication Engineering (IOSR-JECE), Vol. 8, Issue 4, 2013.

[4] Sambaraju, Rakesh. "16-QAM Photonic Vector Modulator for Gigabit Wireless Links." IEEE Journal on Selected Areas in Communications, 2013

[5] Liang Zhang, Xiaofeng Hu, Tao Wang, Qi Liu, Yikai Su," Proposal of A Star-16QAM System Based on Intersymbol Interference (ISI) Suppression and Coherent Detection" Proc. of SPIE, VOL. 7514 5140V-1, 2009

[6] Abdulamir Ali, Werner Rosenkranz, and Jochen Leibrich, "Design considerations and performance comparison of high-order modulation formats using OFDM", IEEE 2012.

[7] Guoying Zhang, Marc De Leenheer, Annalisa Morea, Biswanath Mukherjee, "A survey on OFDM-based electric core optical networking",IEEE 2013.

[8] Sarangi Devasmitha Dissanayake, Jean Armstrong, "Comparison of ACO-OFDM, DCO-OFDM and ADO-OFDM in IM/DD systems", IEEE 2013.

[9] Chin - Sean Sum, Fumihide Kojima, Hiroshi Harada, "Performance analysis of a multi-hop IEEE 802.15.4g OFDM system in multiPHY layer network", IEEE 2013.

[10] Ramon Casellas, Raul Munoz, Josep M. Fabrega, Michela Svaluto Moreolo, Ricardo Martinez, Lei Liu, Takehiro Tsuritani, Itsuro Morita, "Design and experimental validation of a GMPLS/PCE control plane for elastic CO-OFDM optical networks",IEEE 2013.

[11] Xiang Li, Wen-De Zhang, Arokias Wami Alphones, Changyuan $\mathrm{Yu}$, Zhaowen $\mathrm{Xu}$, "Channel equalisation in optical OFDM systems using independent component analysis", IEEE 2014.

[12] Gnauck A.H. et al., "Generation and transmission of 21.4-Gbaud PDM 64-QAM using a high-power DAC driving a single I/Q modulator," Technical Digest of Optical Fiber Communication Conference, PDPB2, 2011. 